\title{
Research Paper: \\ Predicting Preventive Covid-19 Disease Behaviors Based on Health Literacy With the Mediating Role of Media and Social Responsibility in Adults Living in Ahvaz
}

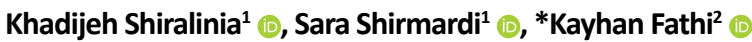

1. Department of Counseling, Faculty of Education and Psychology, Shahid Chamran University of Ahvaz, Ahvaz, Iran

2. Department of Psychology, Institute for Higher Education ACECR, Khuzestan, Iran.

\begin{tabular}{|l|l|l}
$\begin{array}{c}\text { Use yur devic to scan } \\
\text { and read the article online }\end{array}$ & $\begin{array}{l}\text { Citation Shiralinia Kh, Shirmardi S, Fathi K. Predicting Preventive Covid-19 Disease Behaviors Based on Health Literacy With the Medi- } \\
\text { ating Role of Media and Social Responsibility in Adults Living in Ahvaz. Jundishapur Journal of Medical Sciences. 2021; 20(4):390-399. } \\
\text { https://doi.org/10.32598/JSMJ.20.4.2619 }\end{array}$ \\
doilhttps://doi.org/10.32598/JSMJ.20.4.2619
\end{tabular}

Received: 22 Mar 2021

Accepted: 25 Jul 2021

Available Online: 01 Oct 2021

Keywords:

Health literacy,

COVID-19 disease prevention behaviors, Media, Social responsibility

\section{A B STRACT}

Background and Objectives One of the ways to deal with Covid-19 disease is 19 preventive behaviors that seem to be affected by different factors. Therefore, the aim of the present study was to predict the preventive behaviors of Covid- 19 disease based on health literacy with the mediating role of media information belief and social responsibility.

Subjects and Methods This descriptive and correlational study was performed on 247 adults in Ahvaz in the summer of 2020. The research sample was selected using available sampling and they answered online the questionnaires whose validity and reliability were confirmed.. Data were analyzed using Spss, Amose software, descriptive and analytical statistics.

Results The results showed that the indirect coefficients between believing in media information $(P=0.000$, $\beta=0.04)$ and social responsibility $(P=0.000, \beta=0.11)$ with preventive behaviors of corona disease were significant. The proposed research model also had a good fit (RMSEA $=0.05, P=0.20, X^{2} / d f: 1.63, X^{2}: 1.63$ ). Conclusion The results showed that health literacy is both directly and indirectly related to corona disease prevention behaviors through belief in media information and social responsibility. Therefore, to increase the preventive behaviors of corona disease in individuals, we can use the influence of the media and strengthen social responsibility in individuals.

\section{* Corresponding Author:}

Kayhan Fathi, PhD.

Address: Department of Psychology, Institute for Higher Education ACECR, Khuzestan, Iran

Tel: +98 (916) 3045155

E-Mail: keihan.fathi99@gmail.com 


\section{ييشبينى رفتار هاى بيشكَير انه ابتلا به بيمارى كوويد 19 بر اساس سواد سلامت با نقش ميانجى

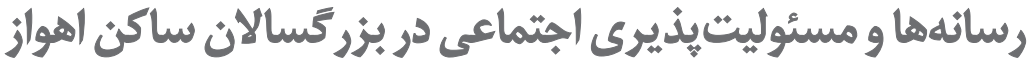

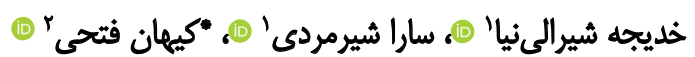

ا. بكروه مشاوره، انشكده علوم تربيتى وروانشناسى، دانشكاه شهيد جمران اهواز، اهواز، ايران. r. أكروه روان شناسى، مؤسسه جهاد دانشكاهى خوزستان، اهوازئ ايران.

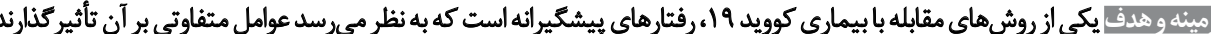

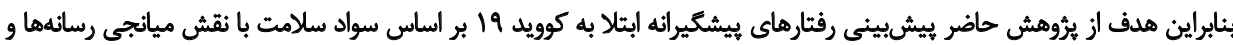
مسئوليتيذيرى اجتماعى است حان.

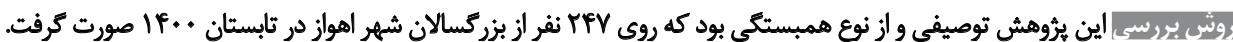

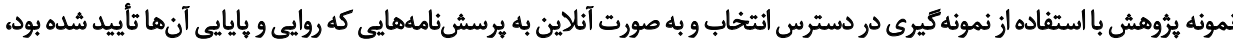

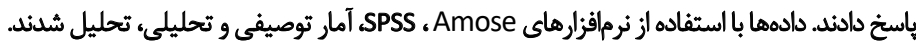

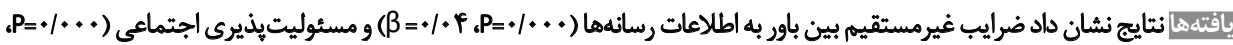

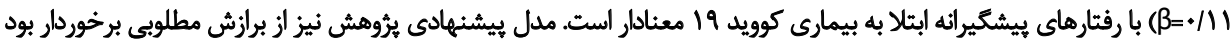

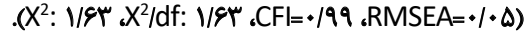

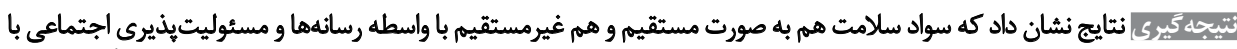

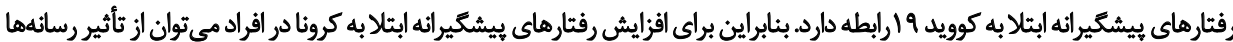

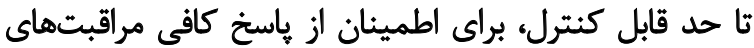

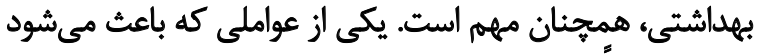

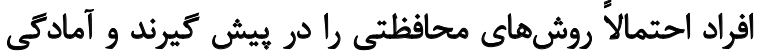

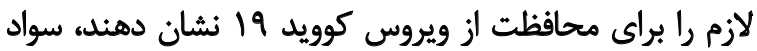
سلامث افراد است [ب]. سواد سلامث به عنوان ادراكات، دانش

از زمان شيوع بيمارى كوويد 9 اميليونها نفر به آن مبتلا

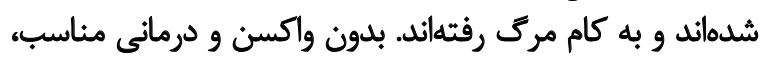

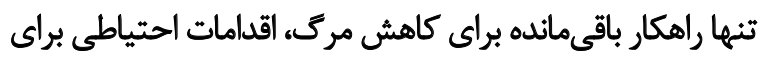

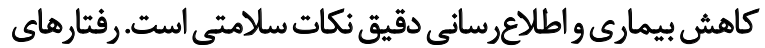

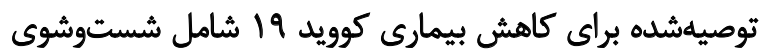

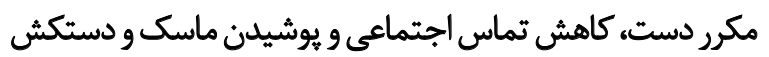

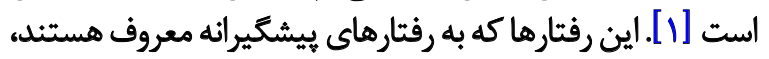
توسط سازمان بهداشت جهانى و مراكز كنترل و و بيشكيري

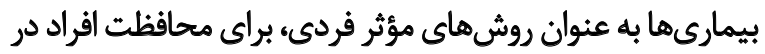

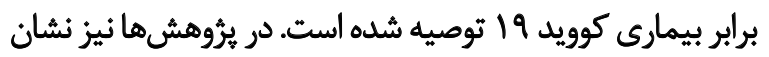




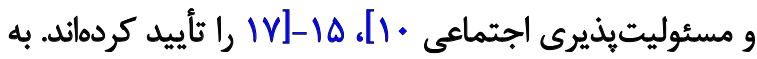

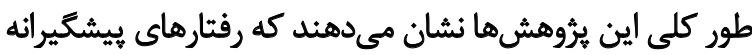

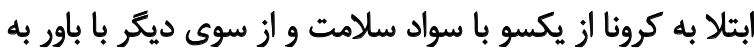

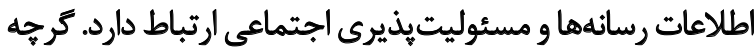

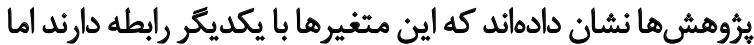

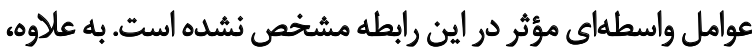

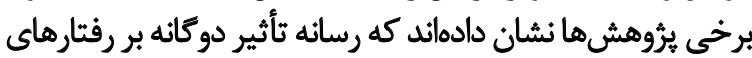

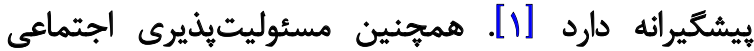
متغيرى است كه نقش اساسى در رفتار اجتماعى دارد [.1.].

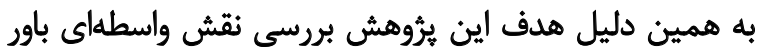

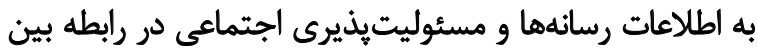

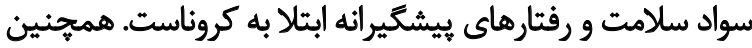

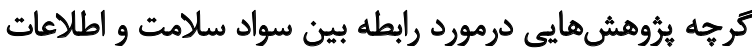

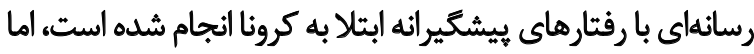

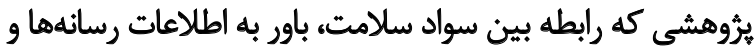

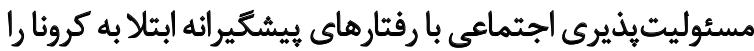

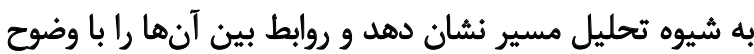

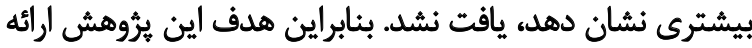

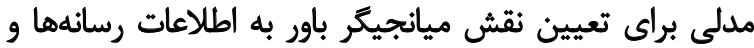

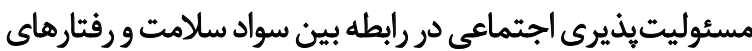

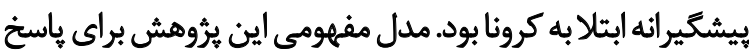
به اين هدف در تصوير شماره الرائه شده است إيت

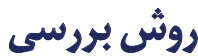

\section{جامعه، نمونه و ووش نمونهيَيرى}

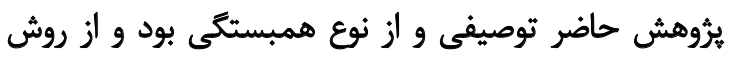

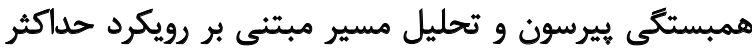

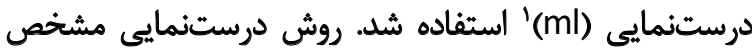

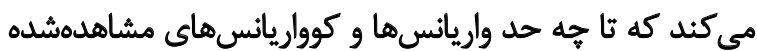

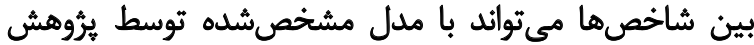

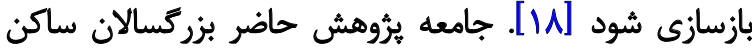

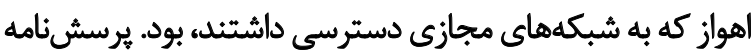

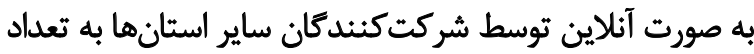

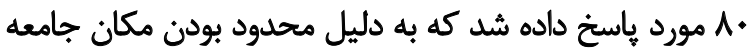

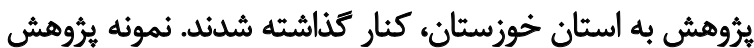

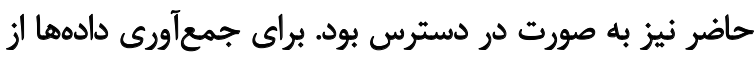

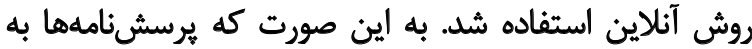

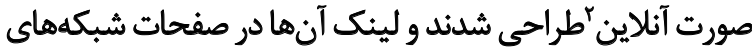

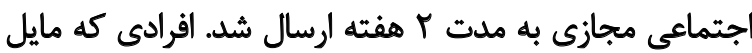

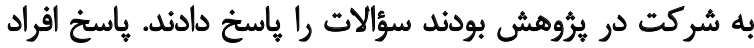

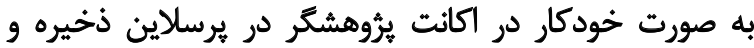

1. Maximum likelihood

2. Link: https://www.digisurvey.net/
و توانايى افراد در درك، دسترسى، ارزيابى و استفاده از اطلاعات

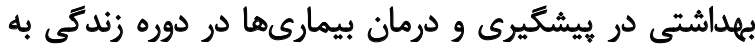

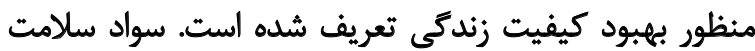

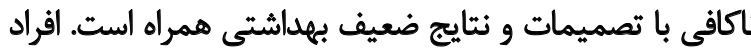

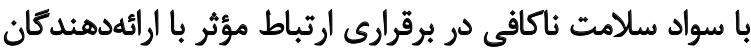

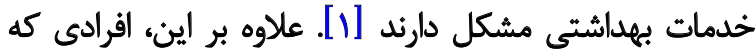

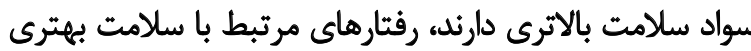

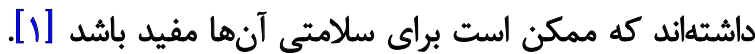

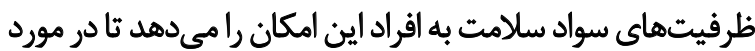

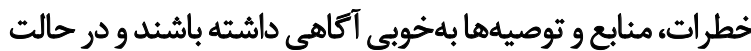

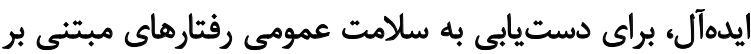

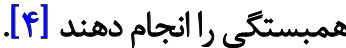

يكى از عوامل كه با افزايش رفتارهاي ييشكيرانه ابتلا به كرونا

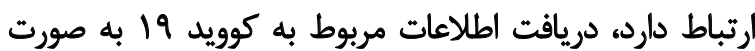

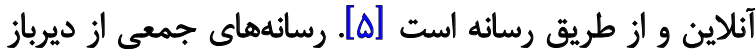

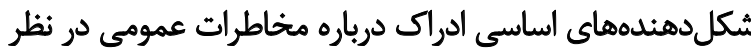

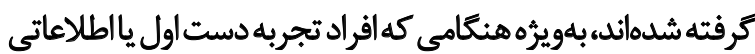

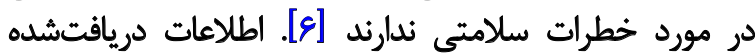

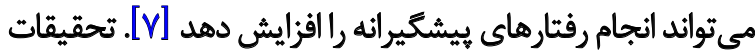

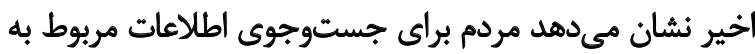

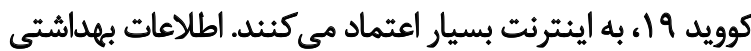

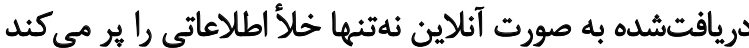

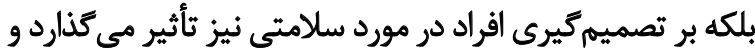

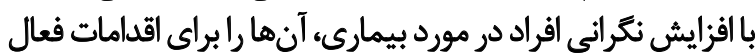

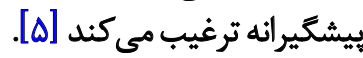

عامل ديكرى كه به نظر مىرسد بر رفتارهاى بيشكَيرانه ابتلا

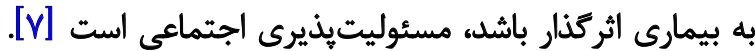

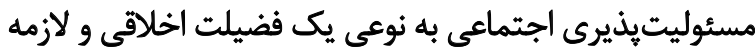

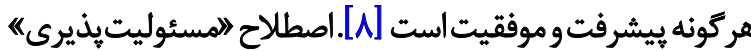

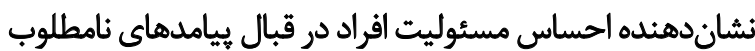

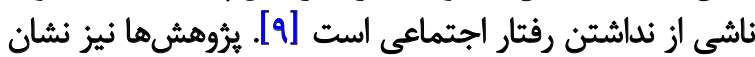

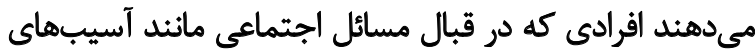

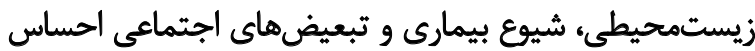

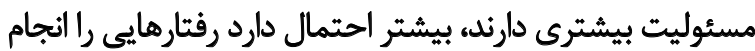

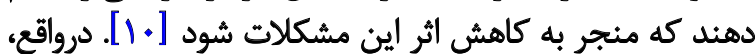

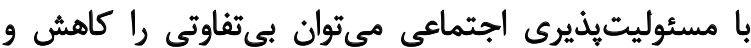
نودوستى را افزايش داد [11].

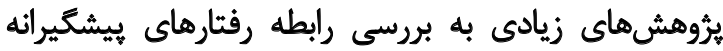

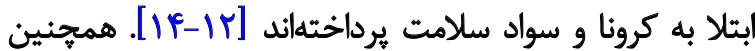

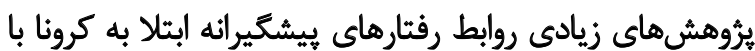

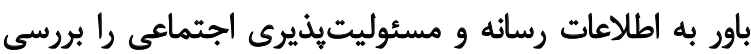

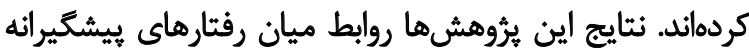

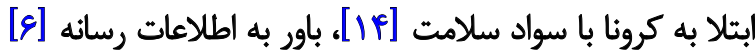




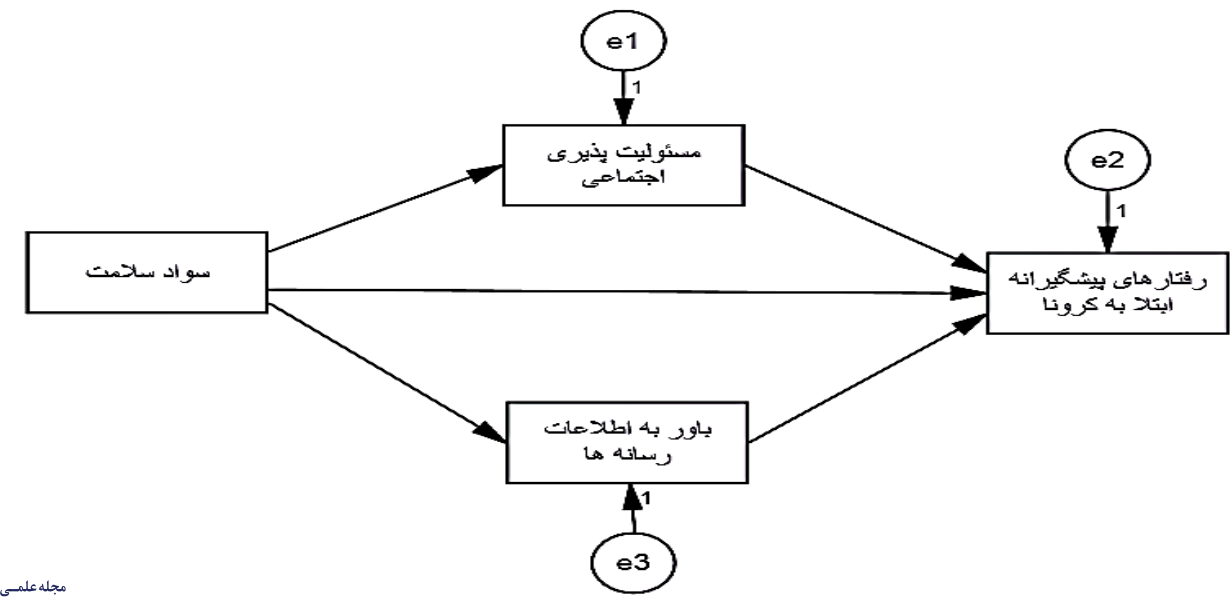

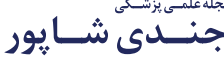

تصوير ا. مدل فرضى نقش ميائجى باور به اطلاعات رسانهها و مسئوليتيذيرى اجتماعى در ارتباط بين سواد سلامت و رفتارهاى بيشكيرائه ابتلا به كرويا

منابع رسانههاى اجتماعى) در اين مقياس شامل اتاق كفتو ألائوى

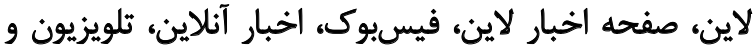

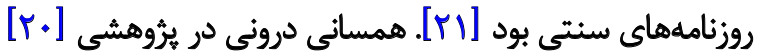

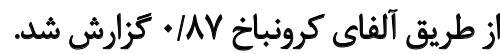

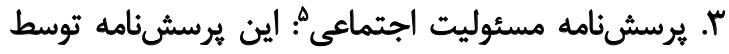

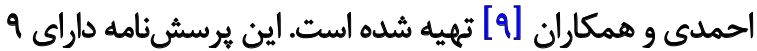

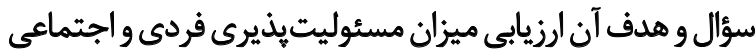

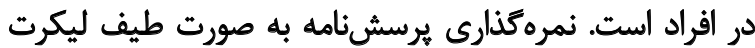
فنقطهاي است. اين يرسشنامه داراى ب بُ بُعد مسئوليتيذئيرى

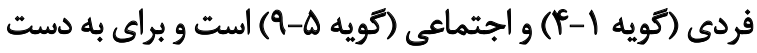
آوردن امتياز كل، مجموع امتيازات مربوط به به تكتئ سؤالات

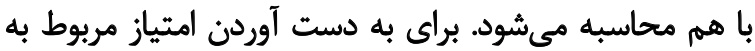

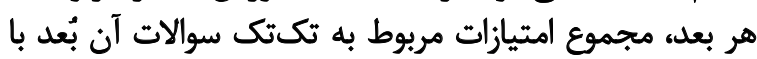

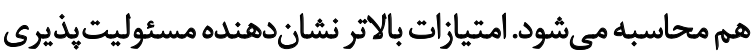

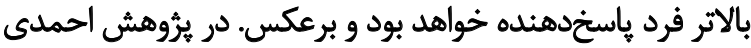

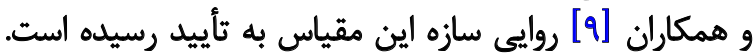

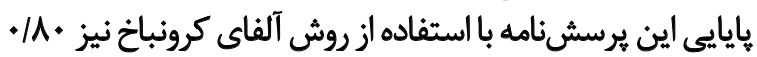

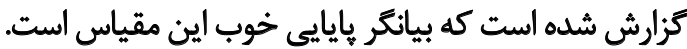

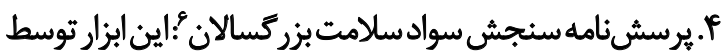

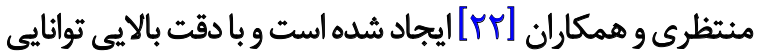

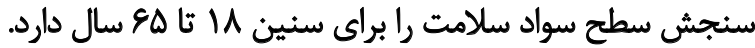

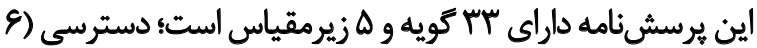

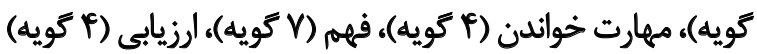

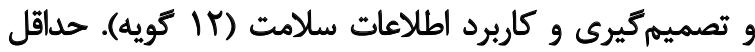

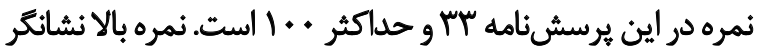

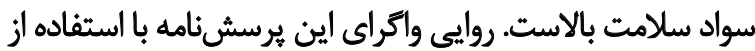

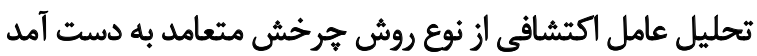

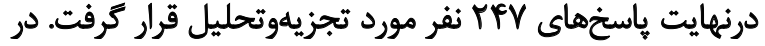

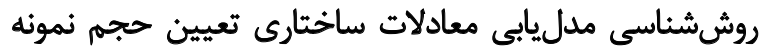

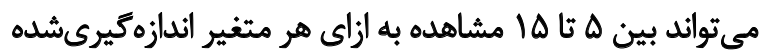

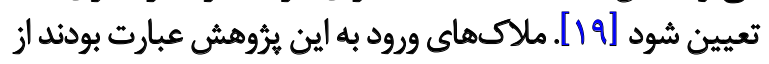

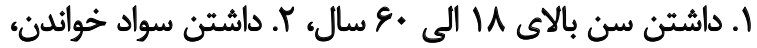

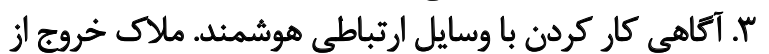

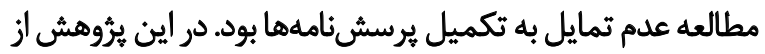

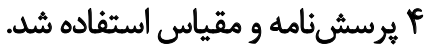

1. برسشنامه رفتارهاى بيشعيرانه ابتلا به بيمارى كوويد

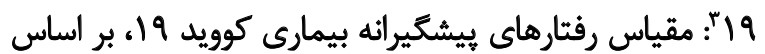

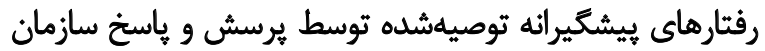

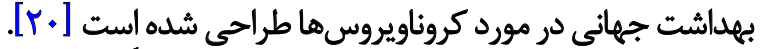

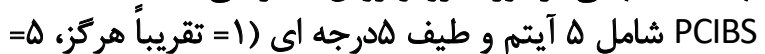

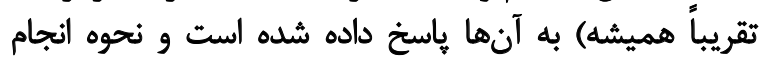

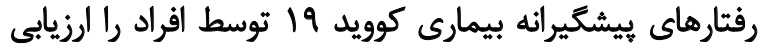

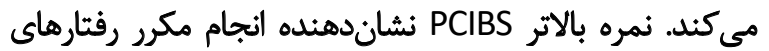

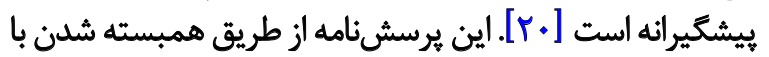

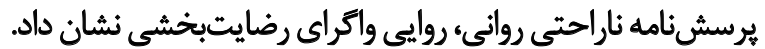

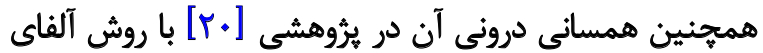
كرونباخ /VV · به دست آمد.

r. مقياس باور به اطلاعات رسانه": مقياس اعتقاد به اطلاعات

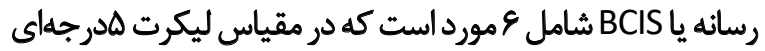

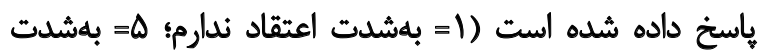

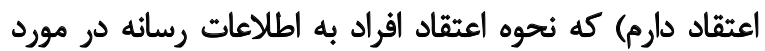

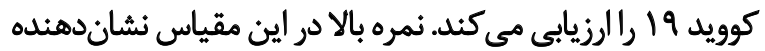

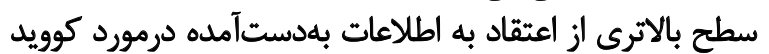

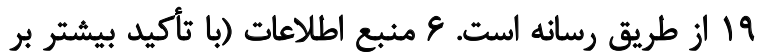




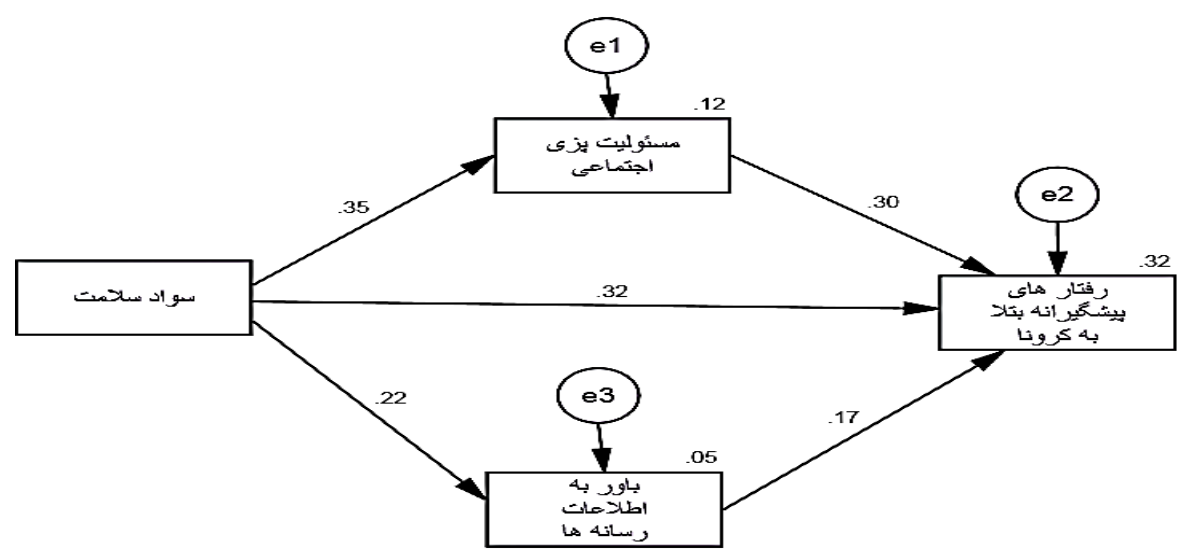

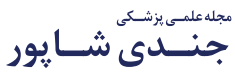

تصوير I. بارهاى استائدارشده مدل نقش ميانجى باور به اطلاعات رسائها و مسئوليت بذيرى اجتماعى در ارتباط بين سواد سلامت و رفتارهاى بيشكيرائه ابتلا به كرونا

جدول شماره r نشان مي دهد، برازي مدل ارائهشده، مطلوب است

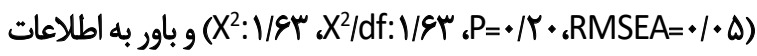
رسائها در ارتباط با سواد سلامت و رفتارهاي بيشئير انه ابتلا به كرونا

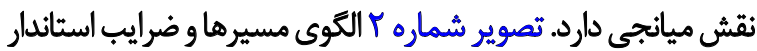

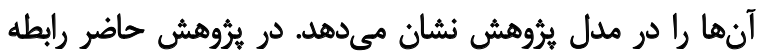

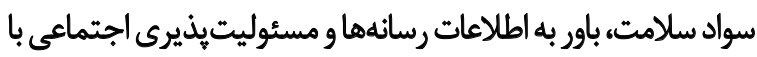
رفتارهاى يُيشير انه ابتلا به كرونا معنادار است.

جدول شماره ب ضرايب ركرسيونى و سطوح معنادارى مدل

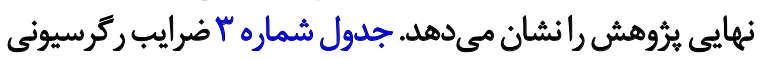

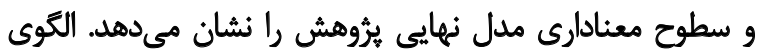

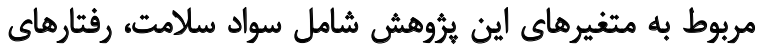

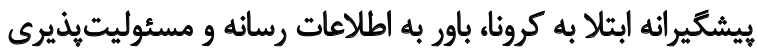

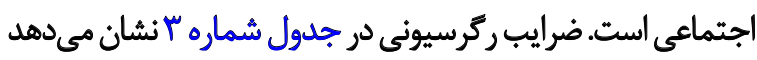

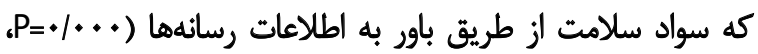

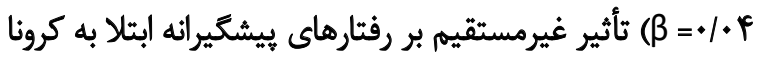

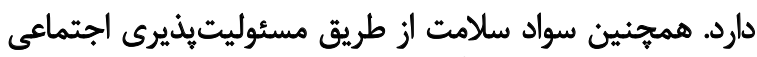
( ثأثير غيرمستقيم بر رفتارهاي بيشئيرانه

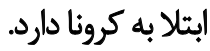

كه نشانكر ارتباط قابل قبول بين متغيرها بود و ساختار هاملي

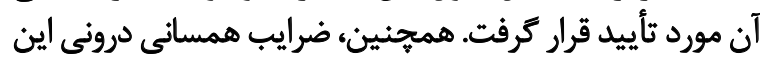

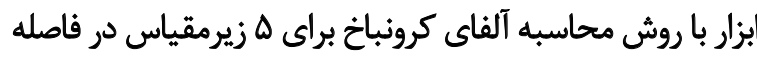

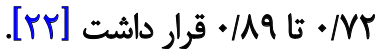

\section{يافتهها}

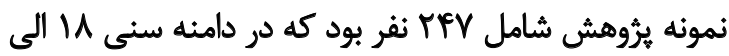

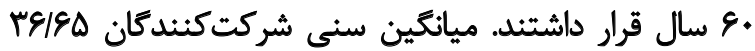

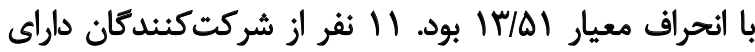

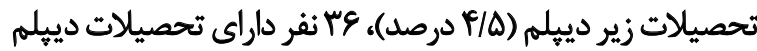
|

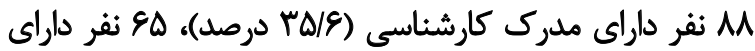

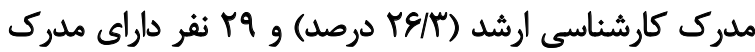

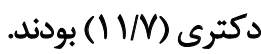

نتايج ماتريس همبستكى در جدول شماره ا نشان مي دهد بين

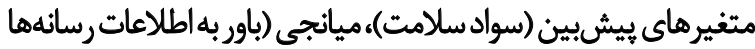

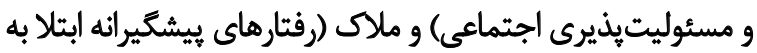

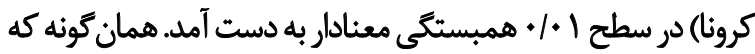

جدول (. آمارههاى توصيفى و ماتريس ضريب همبستكى بين متغيرهاي يُروهش

\begin{tabular}{|c|c|c|c|c|c|c|c|}
\hline$\varepsilon$ & $r$ & $r$ & 1 & كشيدكى & كجى & ميانكين +انحراف معيار & متغير \\
\hline & & & 1 & $T / M$ & $-1 / T r$ & $r y / * \pm T / U$ & ا. رفتارهاى ييشكيرائه ابتلا به كرونا \\
\hline & & 1 & . TA &.$/ M T$ &.$- / 41$ & $\mid q / \cdot . \pm r / q$ & ك. باور به اطلاعات رسانهها \\
\hline & 1 &.$/ 10$ &.$/ \pi$ & $\mathrm{l} / \mathrm{r}$ &.$- / 9$. & TNQE士F/.T & "ا. مسثوليت اجتماعى \\
\hline 1 &.$/ \pi \varphi$ &.$/ \pi$ & .198 & $\cdot M$ &.$- / p 1$ & $V / Q Y \pm \pm I N / N C$ & ". سواد سلامت \\
\hline
\end{tabular}

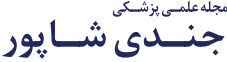




\begin{tabular}{|c|c|c|c|c|c|c|c|}
\hline & & & & & & 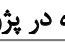 & • شاخص هاي برازندكى مـ \\
\hline RMSEA & CFI & AGFI & GFI & DF & $X^{2} / d f$ & $X^{2}$ & شاخُص هاي برازش مدل \\
\hline .100 &.$/ 99$ & .198 &.$/ 99$ & 1 & V/FT & $V / q T$ & مقادير شاخصها \\
\hline
\end{tabular}

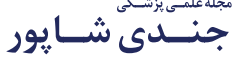

عبارت ديكر، سواد سلامت از طريق ثأثير بر باور به اطلاعات

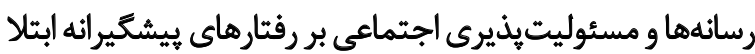

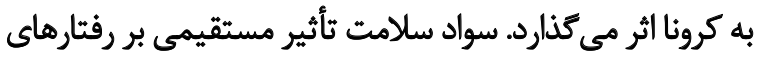

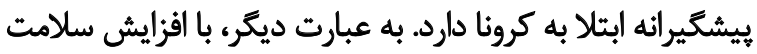

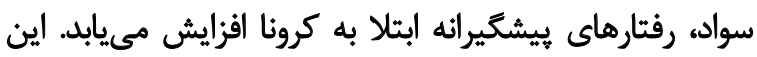

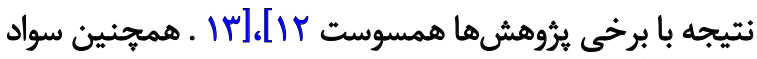

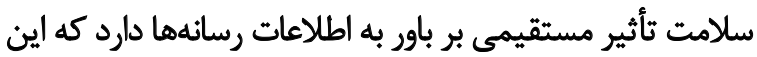

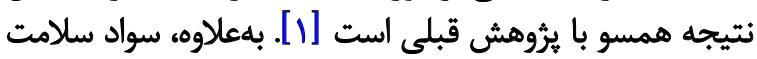

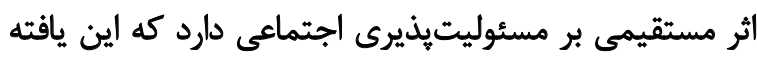

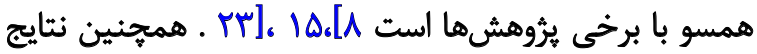

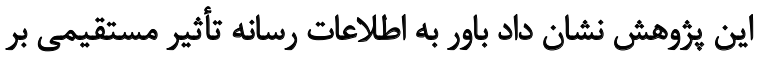

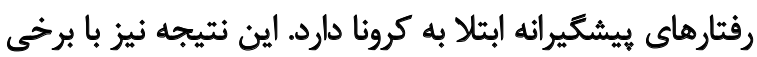

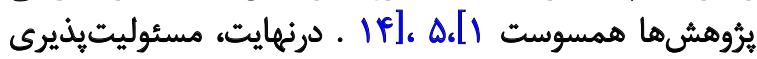

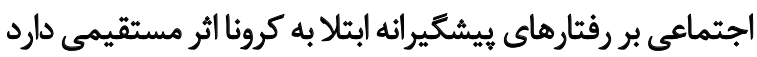

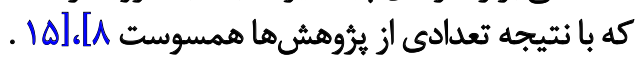
در تبيين نتايج حاصل از يروهش حاضر مى ثوان كفت كه سطح

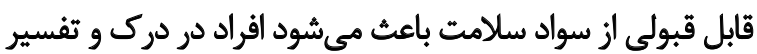

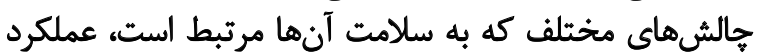

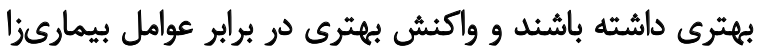

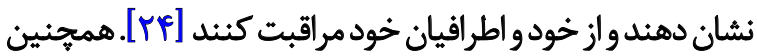

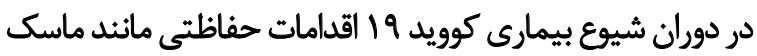

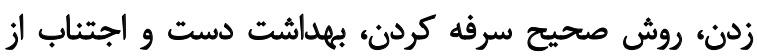

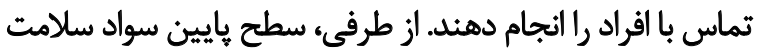

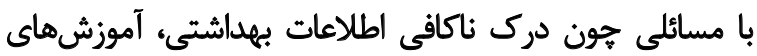

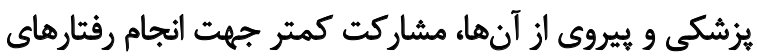

جهت آزمودن اثر غيرمستقيم متغيرها از بوت استراب استفاده

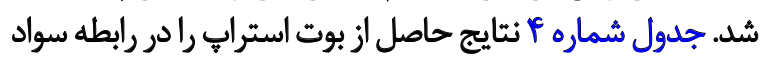

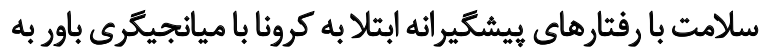

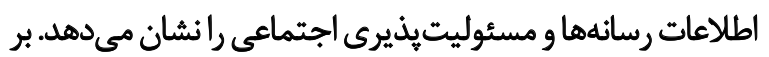

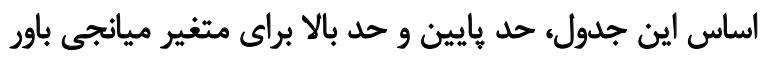

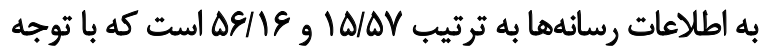

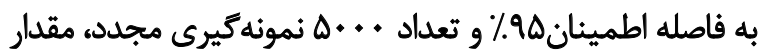

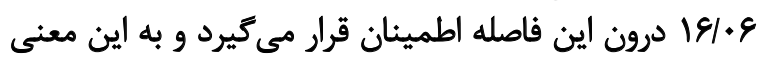

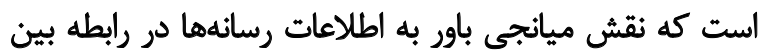

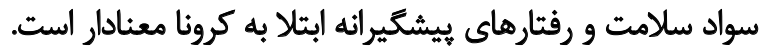

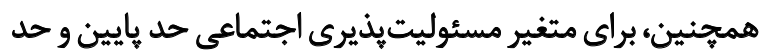

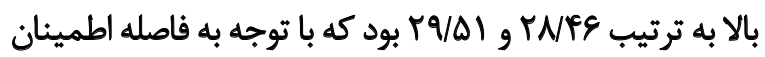

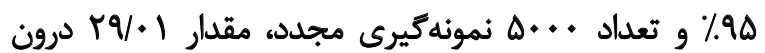

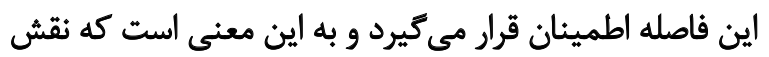

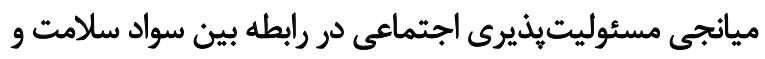
رفتارهاي بيشكيرانه ابتلا به كرونا معنادار است.

ث

هدف اين ثيروهش بررسى نقش ميانجى باور به اطلاعات

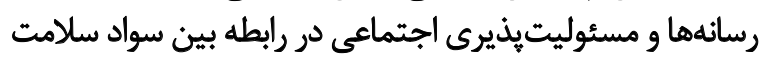

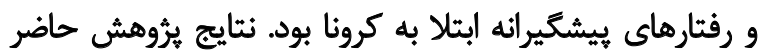

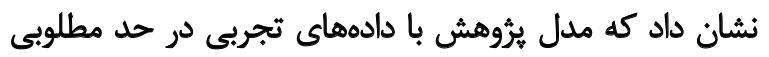

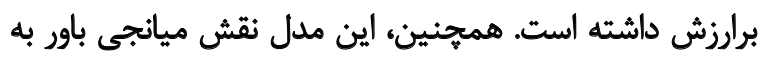

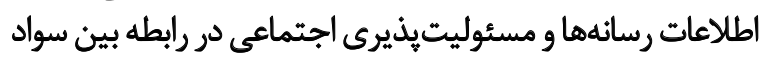

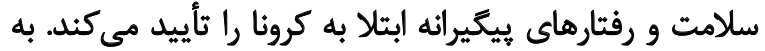

جدول س. ضرايب ركرسيونى و سطوح معنادارى مدل نهايى ثروهش

\begin{tabular}{|c|c|c|c|}
\hline تأثير كذارى & سطح معنادارى & ضريب تأثير & رابطه \\
\hline مستقيم & 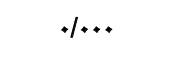 &.$/ 4 \Delta$ & سواد سلامت ؟ مستوليتيذيرى اجتماعى \\
\hline مستقيه & $\%$ &.$/ 4 r$ & سواد سلامت ـ باور به اطالاعات رسانهها \\
\hline مسثقيم & $+\ldots$ & . & سواد سلامت ـ ـرفتارهاى ييشكرانه إبتلا به كرونا \\
\hline مستقيم & $\%$ &.$/ \mu$ & مستوليت ـ ـرفتارهاي ييكيرانه ابتلا به كرونا \\
\hline مسثقيم & $h$ &.$/ 18$ & باور به اطلاعات رسائهها ـ ـرفتارهاى بيشكراثه ابتلا به كرونا \\
\hline غيرمستقميمي & $\%$ & .1 .4 & 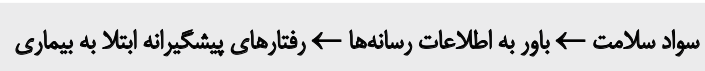 \\
\hline غيرمستقيه & $\%$ &.$M$ & 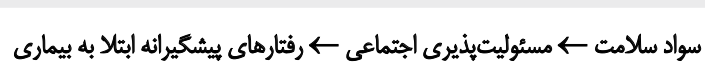 \\
\hline
\end{tabular}

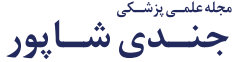


جدول ع. نتايج آزمون بوت استراب (T. T) براى بررسى مسير ميانجى

\begin{tabular}{|c|c|c|c|c|c|c|}
\hline \multirow[t]{2}{*}{ تصميم:ميرى } & \multirow[t]{2}{*}{ حد بالا } & \multirow{2}{*}{ مثغير وابست هايين } & \multicolumn{2}{|c|}{ بوت استراب } & \multicolumn{2}{|c|}{ مسير واسطداى } \\
\hline & & & & & متغير مياثجي & متغير مستقل \\
\hline تأييد اثر غير مستقيم & $\mid S / \Delta S$ & $1 \Delta / \Delta \gamma$ & 191.8 & رفتارهاي يبكيروانها ابتلال & باور به اطلاعات رسانهها & سواد سلامت \\
\hline تأييد اثر غيرمستقيم & rVAI & TWES & rq.1 & رفتارهاى يبيكّراته ابتلا & مستوليتيذيرى اجتماعى & سواد سلامت \\
\hline
\end{tabular}

رفتارهاى اجتماعى درست، بايد آكاهى و سواد افراد از رفتارهاى

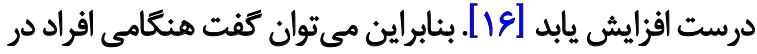

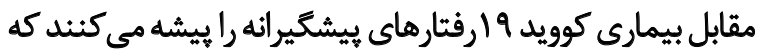

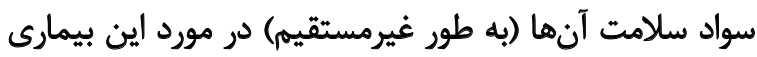

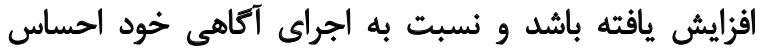

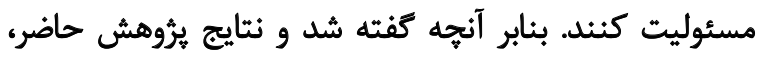

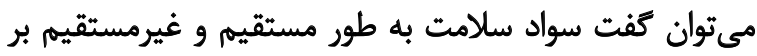
رفتارهاي بيشَّيرانه ابتلا به كرونا اثر مي كذارد.

\section{نتيجليَّى}

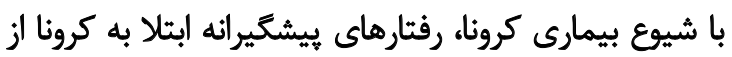

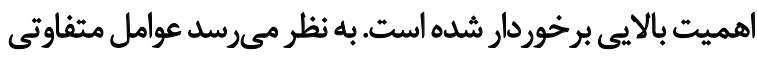

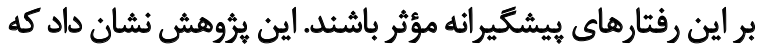

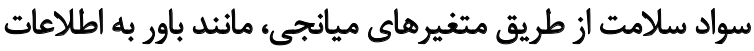

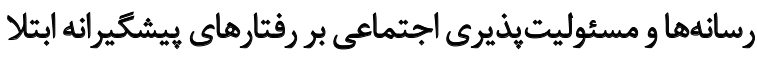

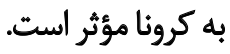

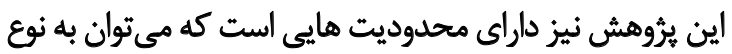

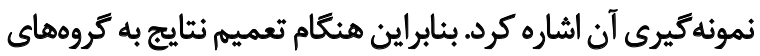

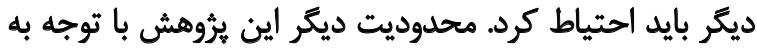

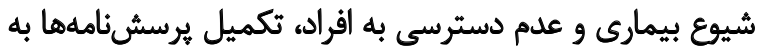

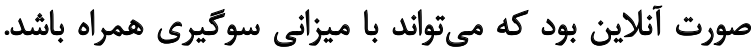

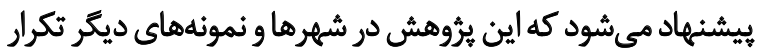

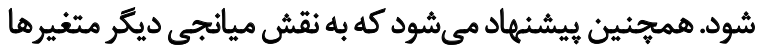

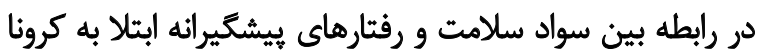

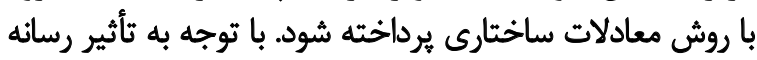

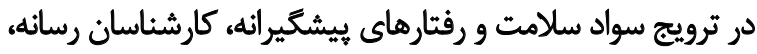

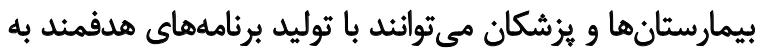

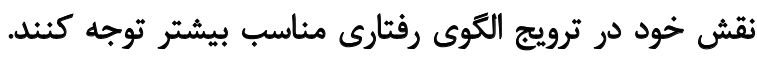

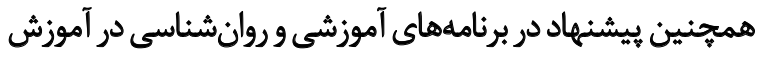

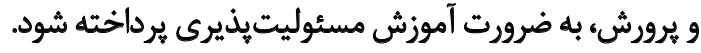

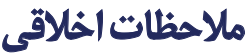

\section{يبيروى أز الصول اخلاق يؤهش}

اين ثُروهش مورد تاييد كميته اخلاق دانشعاه شهيد جمران
بيشخيرانه، تشخيص ديرهنگام بيمارىها، ناتوانى در مهارتهاى

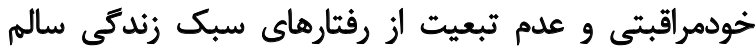

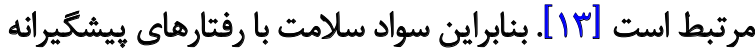

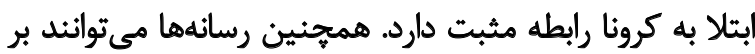

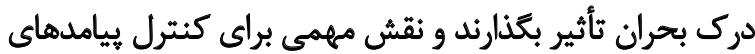

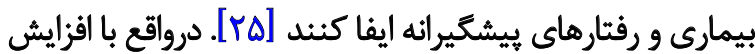

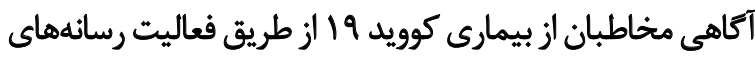

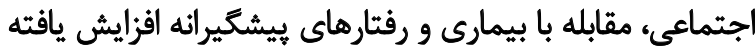

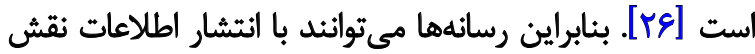

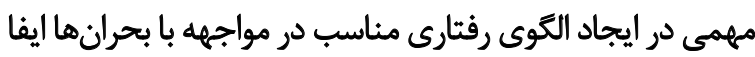

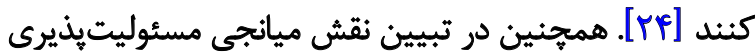

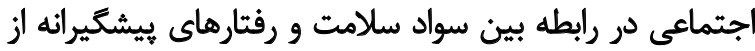

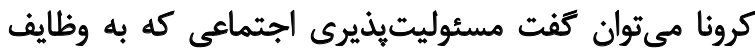

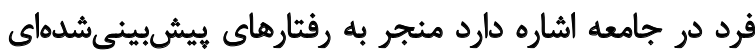

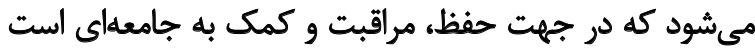

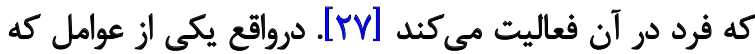

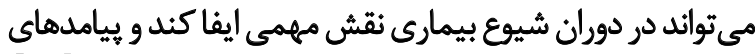

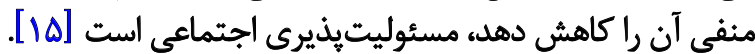

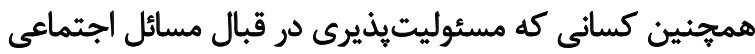

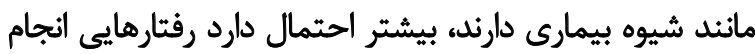

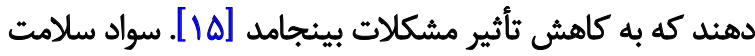

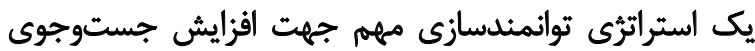

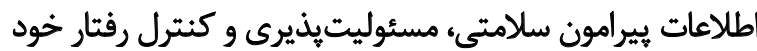

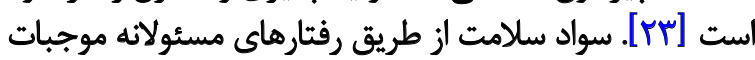

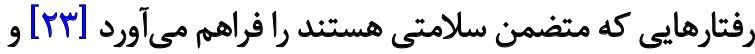

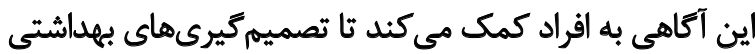

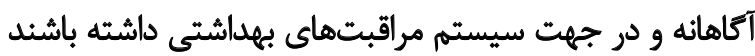

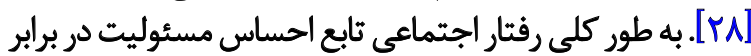

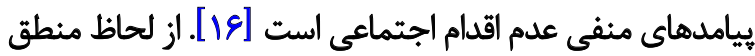

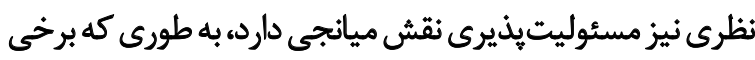

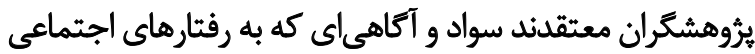

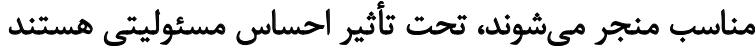

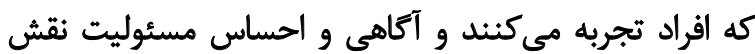

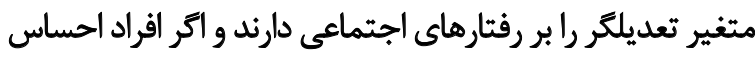

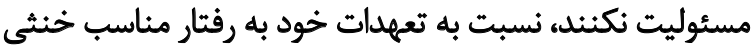
خواهند بود. بهعلاوه قبل از تمركز بر مسئوليتها هنيت نئام ترويج 
اهواز قرار كرفته است )كد اخلاق:ACE/1400.2.24.14542 (SCU.AC.IR

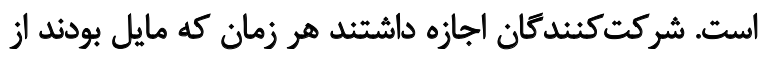

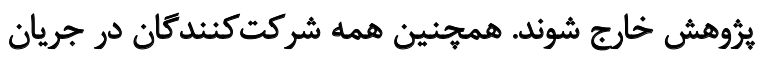

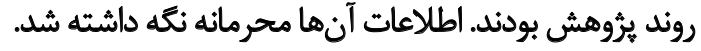

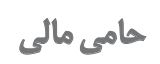

اين تحقيق هيجحونه كمك مالى از سازمانهاي تأمين مالى در

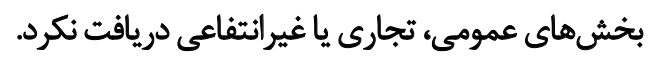

$$
\text { مشاركت ثويسندكان }
$$

تمام نويسندگان در طراحي، اجرا و نكارش همه بخشهاي

ئروهش حاضر مشاركت داشتهاند.

تعارض مثاقع

بنابر اظهار نويسندكان اين مقاله تعارض منافع ندارد. 


\section{Refrences}

[1] Zhao E, Wu Q, Crimmins EM, Ailshire JA. Media trust and infection mitigating behaviors during the COVID-19 pandemic in the USA. BMJ Glob Health. 2020; 5(10):e003323. [DOI:10.1136/bmjgh-2020-003323] [PMID] [PMCID]

[2] Nguyen TT, Le NT, Nguyen MH, Pham LV, Do BN, Nguyen HC, et al. Health literacy and preventive behaviors modify the association between pre-existing health conditions and suspected COVID-19 symptoms: A multi-institutional survey. Int J Environ Res Public Health. 2020; 17(22):8598. [DOI:10.3390/ijerph17228598] [PMID] [PMCID]

[3] Toussaint LL, Cheadle AD, Fox J, Williams DR. Clean and contain: Initial development of a measure of infection prevention behaviors during the COVID-19 pandemic. Ann Behav Med. 2020; 54(9):619-25. [DOI:10.1093/abm/kaaa064] [PMID] [PMCID]

[4] Sentell T, Vamos S, Okan O. Interdisciplinary perspectives on health literacy research around the world: more important than ever in a time of COVID-19. Int J Environ Res Public Health. 2020; 17(9):3010. [DOI:10.3390/ijerph17093010] [PMID] [PMCID]

[5] Li S, Feng B, Liao W, Pan W. Internet use, risk awareness, and demographic characteristics associated with engagement in preventive behaviors and testing: Cross-sectional survey on COVID-19 in the United States. J Med Internet Res. 2020; 22(6):e19782. [DOI:10.2196/preprints.19782] [PMID] [PMCID]

[6] Oh SH, Lee SY, Han Ch. The effects of social media use on preventive behaviors during infectious disease outbreaks: The mediating role of self-relevant emotions and public risk perception. Health Commun. 2021; 36(8):972-81. [DOI:10.1080/10410236.2020.1 724639] [PMID]

[7] Chang KC, Strong C, Pakpour AH, Griffiths MD, Lin CY. Factors related to preventive COVID-19 infection behaviors among people with mental illness. J Formos Med Assoc. 2020; 119(12):1772-80. [DOI:10.1016/j.jfma.2020.07.032] [PMID] [PMCID]

[8] Soroush M. [Personal and social responsibility, altruism and social trust: A study of adolescents in Shiraz (Persian)]. J Appl Sociol. 2012; 23(2):193-211. https://jas.ui.ac.ir/article_18267.html

[9] Ahmadi S, Mirfardi A, Zarei Gh. [An investigation of the relationship between responsibility and attitude towards saving water (Persian)]. J Appl Sociol. 2012; 24(2):185-200. https://jas.ui.ac.ir/ article_18309.html

[10] Han H, Lee S, Kim JJ, Ryu HB. Coronavirus Disease (COVID-19), traveler behaviors, and international tourism businesses: Impact of the Corporate Social Responsibility (CSR), knowledge, psychological distress, attitude, and ascribed responsibility. Sustainability. 2020; 12(20):8639. [DOI:10.3390/su12208639]

[11] Kalantari S, Adibi M, Rabbani R, Ahmadi S. [An investigation on apathy and altruism in urban society of Iran (Persian)]. Daneshvar Raftar. 2007; 14(22):27-35. https://www.sid.ir/fa/journal/ViewPaper.aspx?id=68177

[12] Khazaee-Pool M, Shahrousvand Sh, Naghibi SA. [Predicting Covid-19 preventive behaviors based on health belief model: An Internet-based study in Mazandaran province, Iran (Persian)]. J Mazandaran Univ Med Sci. 2020; 30(190):56-66. http://jmums. mazums.ac.ir/article-1-15530-en.html
[13] Aghamolaei T, Hosseini Z, Hosseini F, Ghanbarnejad A. [The relationship between health literacy and health promoting behaviors in students (Persian)]. J Prev Med. 2016; 3(2):36-43. http://jpm.hums.ac.ir/article-1-173-fa.html

[14] Li Sh, Cui G, Kaminga AC, Cheng S, Xu H. Associations between health literacy, eHealth literacy, and COVID-19-related health behaviors among Chinese college students: Cross-sectional online study. J Med Internet Res. 2021; 23(5):e25600. [DOI:10.2196/25600] [PMID] [PMCID]

[15] Han H, Hwang J. Norm-based Loyalty Model (NLM): Investigating delegates' loyalty formation for environmentally responsible conventions. Int J Hosp Manag. 2015; 46:1-14. [DOI:10.1016/j.ijhm.2015.01.002]

[16] De Groot JIM, Steg L. Morality and prosocial behavior: The role of awareness, responsibility, and norms in the norm activation model. J Soc Psychol. 2009; 149(4):425-49. [DOI:10.3200/ SOCP.149.4.425-449] [PMID]

[17] Oliver RL. Satisfaction: A behavioral perspective on the consumer: A behavioral perspective on the consumer. $2^{\text {nd }}$ ed. Routledge; 2014. https://books.google.com/ books? $i d=$ TzrfBQAAQBAJ $\&$ printsec=

[18] Skrondal A, Rabe-Hesketh S. Generalized latent variable modeling: Multilevel, longitudinal, and structural equation models. $1^{\text {st }}$ edition. Boca Raton, Florida: Chapman and Hall/CRC Publlication; 2004. https://books.google.com/ books?id=e1DcsgEACAAJ \&dq=

[19] Hooman HA. [Structural equation modeling with Lisrel application (Persian)]. $1^{\text {st }}$ ed. Tehran: Samt; 2005. http://opac.nlai. ir/opac-prod/bibliographic/2060723

[20] World Health Organization. Coronavirus Disease 2019 (COVID-19) situation report - 95 [Internet]. 2020 [Updated 2020 April 24]. Available from: https://www.who.int/docs/defaultsource/coronaviruse/situation-reports/20200424-sitrep95-covid-19.pdf

[21] Chang KC, Hou WL, Pakpour AH, Lin CY, Griffiths MD. Psychometric testing of three COVID-19-related scales among people with mental illness. Int J Ment Health Addict. 2020; July. [DOI:10.1007/s11469-020-00361-6] [PMID] [PMCID]

[22] Montazeri A, Tavousi M, Rakhshani F, Azin SA, Jahangiri K, Ebadi M, Naderimagham Sh, et al. [Health Literacy for Iranian Adults (HELIA): Development and psychometric properties (Persian)]. Payesh. 2014; 13(5):589-99. http://payeshjournal. ir/article-1-279-en.html

[23] Sørensen K, Brand H. Health literacy--a strategic asset for corporate social responsibility in Europe. J Health Commun. 2011; 16(Suppl 3):322-7. [DOI:10.1080/10810730.2011.6060 72] [PMID]

[24] Peyvand M, Kargar Sh, Hajizade F. The role of health literacy promotion in epidemic control corona 19. J Health Lit. 2020; 5(1):9-11. [DOI:10.22038/JHL.2020.47609.1109]

[25] McKinnon S, Gorman-Murray A, Dominey-Howes D. Disasters, queer narratives, and the news: How are LGBTI disaster experiences reported by the mainstream and LGBTI media? J Homosex. 2017; 64(1):122-44. [DOI:10.1080/00918369.2016. 1172901] [PMID] 
[26] Ayeneh M, Hosseinpour M. [Investigating the influence of factors effective in using social media on improving e-health literacy of citizens in crisis (Persian)]. J Health Biomed Inform. 2021; 8(1):84-93. http://jhbmi.ir/article-1-545-en.html

[27] Hashemi SA, Hamrahi M. [Social responsibility (Persian)]. Paper presented at: $3^{\text {rd }}$ International Conference on Modern Research's in Management, Economics \& Accounting. 15 March 2016; Istanbul, Turkey. https://civilica.com/doc/554689/

[28] Spencer M, Kemp N, Cruickshank V, Otten C, Nash R. An international review to characterize the role, responsibilities, and optimal setting for health literacy mediators. Glob Pediatr Health. 2021; 8. [DOI:10.1177/2333794X211025401] [PMID] [PMCID] 